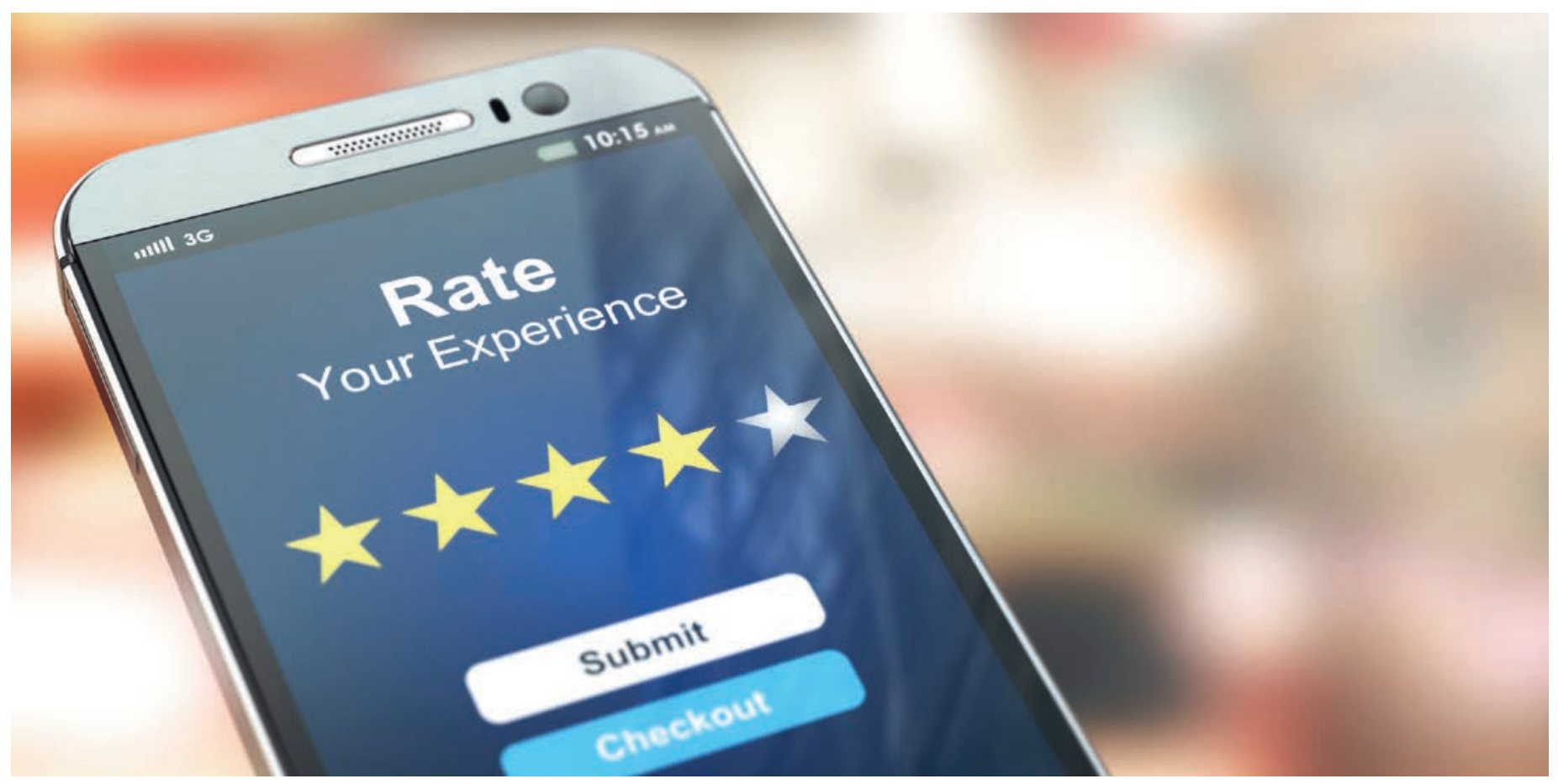

\title{
Aspects juridiques concernant les évaluations sur internet
}

\author{
Michael Barnikol ${ }^{a}$, Reinhold Sojer ${ }^{b}$, Fabian Röthlisberger ${ }^{c}$ \\ ${ }^{a}$ Dr iur., juriste au Service juridique, $\mathrm{FMH}^{\circ}{ }^{\mathrm{b}}$ Dr rer. biol. hum., chef de la division Numérisation / eHealth, FMH; \\ ${ }^{c}$ collaborateur scientifique, chef suppléant de la division Numérisation / eHealth, FMH
}

En raison de leur popularité croissante dans d'autres domaines et de l'attention accrue portée à la satisfaction des patients et à la qualité de traitement, les médecins doivent accorder davantage d'attention au thème des évaluations en ligne. La question de savoir dans quelle mesure ces évaluations peuvent réellement refléter la qualité de la consultation médicale fait l'objet d'avis divergents dans la littérature. D'une part, des études démontrent qu'il n'existe pas de corrélation entre les évaluations de patients et les critères médicaux objectifs relatifs au résultat médical [1, 2]. D'autre part, les patients sont tout à fait en mesure d'évaluer les paramètres tels que l'infrastructure, l'organisation ou l'amabilité, ces facteurs contribuant aussi à la qualification de «bon médecin» [3]. En cas d'évaluations en ligne inappropriées, les médecins se trouvent face à d'importants défis (juridiques), notamment en raison des réglementations spécifiques à la profession comme p. ex. l'obligation de respect du secret professionnel [4-6]. Souvent, ils ne peuvent pas répondre aux auteurs, les évaluations étant généralement déposées sous couvert de l'anonymat. Le droit de réponse n'est pas non plus d'un grand secours, car le médecin devrait alors se servir d'informations contenues dans le dossier médical. Sans consentement du patient ou levée du secret médical par l'autorité cantonale de surveillance, cela représenterait une violation du secret médical.

\section{Les patients font encore rarement usage de ces portails d'évaluation.}

En Suisse, on dispose avec Google et des sites tels que medicosearch.ch, okdoc.ch, docapp.ch ou tondocteur. ch de différentes possibilités pour évaluer les médecins [7]. Cependant, les patients font encore rarement usage de ces portails d'évaluation. Dans le cadre du baromètre de la cybersanté (eHealth Barometer) 2019, la FMH a interrogé la population au sujet de l'utilisation des portails d'évaluation des médecins. Seuls $2 \%$ des 
sondés ont indiqué avoir évalué leur médecin au cours de l'année dernière ${ }^{1}$. Près de trois quarts des personnes interrogées ont par ailleurs indiqué ne pas considérer les évaluations en ligne comme un critère important lors du choix du médecin. Les recommandations personnelles semblent toujours être plus pertinentes que des informations publiques impersonnelles $[8,9]$. Malgré la faible utilisation actuelle, il faut partir du principe que le nombre d'évaluations en ligne augmentera à l'avenir en raison du désir d'obtenir une transparence maximale.

\section{Aspects juridiques des portails d'évaluation en ligne}

Les portails d'évaluation offrent généralement de nombreuses indications sur le cabinet ou le médecin. Sur le plan juridique, la protection des données est donc essentielle pour leur appréciation. La réglementation figure dans la loi sur la protection des données (LPD) qui s'applique lorsque des données personnelles sont traitées, c'est-à-dire toutes les données qui se rapportent à une personne identifiée ou identifiable (art. 2, al. 1, et art. 3, let. a, e et f, LPD). La publication d'informations et d'évaluations concernant les médecins sur internet en fait notamment partie ${ }^{2}$.

Pour l'évaluation de médecins, il faut donc tenir compte des principes relatifs au traitement de données personnelles selon l'art. 4 et les art. 12ss LPD. Le traitement ne doit notamment pas porter atteinte à la personnalité de la personne concernée: il doit être effectué conformément aux principes de la bonne foi et de la proportionnalité, et aucune donnée ne peut être traitée contre la volonté expresse de la personne concernée (art. 12, al. 1 et al. 2, let. a et b, LPD, en relation avec l'art. 4 LPD). Il peut s'agir d'une atteinte à la personnalité si le droit de disposer de ses données personnelles (droit à l'autodétermination en matière d'information) ou d'autres droits de la personnalité sont menacés ${ }^{3}$.

C'est p. ex. le cas si l'évaluation se fait contre la volonté explicite du médecin concerné, qu'elle contient des allégations de fait manifestement fausses ou présente un contenu diffamatoire ou dénigrant ${ }^{4}$.

Cependant, un traitement de données portant atteinte à la personnalité peut être justifié dans certains cas. Il peut être justifié par le consentement de la victime, par un intérêt prépondérant privé ou public, ou par la loi (art. 13, al. 1, LPD)

Aux droits des médecins concernés - en premier lieu le droit à l'autodétermination en matière d'information s'opposent donc les intérêts économiques des exploitants des plateformes d'évaluation, l'intérêt à la libre circulation des données et la liberté d'opinion des utilisateurs qui déposent les évaluations. En Suisse, il n'existe pas encore de jurisprudence susceptible de fournir une aide à l'orientation pour cette pesée des intérêts. La Cour fédérale de justice allemande (Bundesgerichtshof, BGH) s'est exprimée à ce sujet dans sa jurisprudence - cela eu égard au fait que, selon l'art. 29,

\section{En Suisse, il n'existe pas encore de jurispru-} dence susceptible de fournir une aide à l'orientation pour cette pesée des intérêts.

al. 1, p. 1, n 1 , de la loi fédérale (allemande) sur la protection des données, la collecte et la conservation des données à caractère personnel à des fins de transmission n'est licite que s'il n'y a pas de raison de penser que la personne concernée a un intérêt personnel légitime à exclure la collecte ou conservation desdites données ${ }^{6}$

Dans le cas en question, une dermatologue a saisi la $\mathrm{BGH}$, étant donné que la plateforme d'évaluation Jameda avait affiché de la publicité pour des médecins concurrents sur son profil en ligne. La BGH a constaté que la plateforme d'évaluation s'éloignait ainsi de sa position de fournisseur d'informations et qu'il existait de ce fait pour le médecin "un intérêt légitime à l'exclusion de la conservation de ses données».

Il n'existe en tout cas pas d'intérêt prépondérant pour la diffusion d'évaluations injurieuses ou mensongères. En conséquence, les exploitants de portails interdisent dans leurs conditions d'utilisation de telles déclarations et offrent la possibilité de les leur communiquer afin qu'elles puissent être supprimées. C'est judicieux, car cela permet d'éviter en amont un litige fastidieux et coûteux. Il serait toutefois souhaitable et conforme au droit à l'autodétermination en matière d'information que les médecins qui refusent leur inscription sur un portail d'évaluation aient en tout temps la possibilité de supprimer globalement leur inscription professionnelle (y compris les évaluations négatives, mais aussi les commentaires positifs et d'autres données personnelles). Cela ne remettrait pas en question le modèle commercial des portails d'évaluation et éviterait le traitement de données - en principe illicite selon l'art. 12, al. 2, let. b-qui, dans un nombre considérable de cas, se fait sans le consentement des médecins concernés.

S'il y a atteinte illicite à la personnalité, les médecins concernés peuvent demander à l'exploitant du portail de cesser tout traitement de données et de supprimer ou corriger les données (art. 15, al. 1, LPD, en relation avec l'art. 28, 28a et 28l, CC). Ces prétentions sont dirigées contre toutes les personnes qui ont participé à l’atteinte illicite à la personnalité7. 
7 Rosenthal/Jöhri, op. cit., art. 12 , ch. 10.

8 Un fichier est tout ensemble de données personnelles dont la structure permet de rechercher les données par personne concernée (art. 3, let. g. LPD)

9 Cf. explications du PFPDT sur les plateformes d'évaluation en ligne, op. cit.

\section{Correspondance:}

FMH

Division Service juridique

Elfenstrasse 18

Case postale 300

CH-3000 Berne 15

lex[at]fmh.ch
De plus, la LPD prévoit un droit d'accès vis-à-vis du maître d'un fichier ${ }^{8}$ (art. 8, al. 1, LPD). La notion de «maître du fichier» est large: elle concerne toute personne qui décide du but du fichier et des moyens et méthodes de traitement ainsi que de son contenu, c'est-à-dire de son existence et de sa structure (art. 3, let. i, LPD). On peut donc partir du principe que les administrateurs des plateformes d'évaluation, en tant que maitres des fichiers ${ }^{9}$, sont concernés, et que les médecins concernés disposent donc aussi d'un droit d'accès vis-à-vis de l'exploitant du portail.

\section{Recommandations de la FMH concernant les évaluations en ligne}

Comme les évaluations en ligne peuvent avoir de graves conséquences sur le médecin et son cabinet, la FMH élabore actuellement diverses aides pour ses membres. Outre les aspects juridiques des portails d'évaluation en ligne publiés ici, un guide de communication pour gérer les évaluations en ligne est en cours d'élaboration. Il est par ailleurs prévu de définir des critères de qualité auxquels un portail d'évaluation sérieux doit répondre du point de vue de la FMH. Ces critères visent à sensibiliser les exploitants de portails et à les rendre attentifs à une évaluation équitable des médecins. Les résultats de ces travaux seront publiés en été 2019.

\section{Crédit photo}

๑) Maksym Yemelyanov | Dreamstime.com (scène fictive)

\section{Références}

1 Okike K, Peter-Bibb TK, Xie KC, Okike ON. Association between physician online rating and quality of care. J Med Internet Res. 2016.

\section{Conclusion}

- Les évaluations en ligne des médecins vont augmenter en raison du désir d'établir une transparence maximale.

- Comme la publication des données de médecins correspond à un traitement de données personnelles, c'est la loi sur la protection des données (LPD) qui s'applique.

- Le médecin a droit à ce que des données publiées portant atteinte à sa personnalité soient supprimées ou corrigées.

- Pour I'heure, aucune jurisprudence n'existe en Suisse sur la question des évaluations en ligne de médecins.

- La FMH élabore actuellement des recommandations pour ses membres sous la forme d'un guide de communication. Elle prévoit par ailleurs de définir des critères de qualité à I'intention des exploitants de portails afin de garantir une évaluation équitable des médecins.

2 Daskivich TJ, Houman J, Fuller G, Black JT, Kim HL, Spiegel B. Online physician ratings fail to predict actual performance on measures of quality, value, and peer review. J Am Med Informatics Assoc. 2018.

3 Rothenfluh F, Schulz PJ. Physician rating websites: What Aspects are important to identify a good doctor, and are patients capable of assessing them? A mixed-methods approach including physicians' and health care consumers' perspectives. J Med Internet Res. 2017.

4 Meienberg O. Arzt-Entwertung im Internet. Bull Med Suisses. 2018

5 Meienberg O. Praxisärzte-Rating auf Abwegen. Arzt-Entwertung im Internet. Bull Med Suisses. 2018.

6 Mahler D. FMH sollte sich der Problematik «Ärztebewertungen im Internet» dringlich annehmen! Bull Med Suisses. 2018.

7 Büchi S. Arztbewertung - Wie viele Sterne dürfen's denn sein. SRF [Internet]. 2016; Available from: https://www.srf.ch/sendungen/ puls/gesundheitswesen/arztbewertung-wie-viele-sterne-duerfens-denn-sein

8 McLennan S, Strech D, Meyer A, Kahrass H. Public awareness and use of German physician ratings websites: Cross-sectional survey of four North German cities. Journal of Medical Internet Research. 2017.

9 Burkle CM, Keegan MT. Popularity of internet physician rating sites and their apparent influence on patients' choices of physicians. BMC Health Serv Res. 2015. 